Two rational automorphs $A_{1}$ and $A_{2}$ of $f$ may be called rightequivalent if there exists an integral automorph $I$ of $f$ such that $A_{1} I=A_{2}$. If $\left(T_{1}, U_{1}\right)$ and $\left(T_{2}, U_{2}\right)$ belong to the same set* of solutions of (5), the corresponding automorphs (with $t=T_{i} / m$, $\left.u=U_{i} / m\right)$ are readily seen to be right-equivalent.

McGill University

\title{
A NOTE ON RECURSIVE FUNCTIONS $\dagger$
}

\section{BY S. C. KLEENE}

The notion of a recursive function of natural numbers, which is familiar in the special cases associated with primitive recursions, Ackermann-Péter multiple recursions, and others, has received a general formulation from Herbrand and Gödel. The resulting notion is of especial interest, since the intuitive notion of a "constructive" or "effectively calculable" function of natural numbers can be identified with it very satisfactorily. $\ddagger$ Consider the operation of passing from a function $\rho\left(x_{1}, \cdots\right.$, $\left.x_{n}, y\right)$, such that for each set of values of $x_{1}, \cdots, x_{n}$ the equation $\rho\left(x_{1}, \cdots, x_{n}, y\right)=0$ has solutions for $y$, to the function " $\epsilon y\left[\rho\left(x_{1}, \cdots, x_{n}, y\right)=0\right]$ " of which the least solution is $x_{1}, \cdots, x_{n}$. We have shown that the (general) recursive functions are the functions which are derivable from the primitive recursive functions by one application of this operation and of substitution. $\$$ Herein we note the related result, that the recursive functions are the functions obtainable by repeated applications of the operation just described and of substitution from the three particular functions $x+y$ (sum), $x \cdot y$ (product), $\delta_{y}^{x}$ (Kronecker delta). This result follows from the other by an adaptation of an argument used by Gödel in proof that every

*For definition of set, see Pall, Transactions of this Society, vol. 35 (1933), p. 491; or Dirichlet, Vorlesungen über Zahlentheorie, \$87.

$\dagger$ Presented to the Society, January 1, 1936.

¥ See A. Church, An unsolvable problem of elementary number theory, American Journal of Mathematics, vol. 58 (1936), pp. 345-363, §7.

$\S \mathrm{S}$. C. Kleene, General recursive functions of natural numbers, Mathematische Annalen, vol. 112 (1936), No. 5, IV and V. 
primitive recursive relation is arithmetic, which we now present.* In doing so, we duplicate in part the analysis of primitive recursive functions given by Hilbert and Bernays. $\dagger$ For the notation and terminology, see our cited article.

Definition 1. Let $C$ denote the least class of functions such that $x+y, x \cdot y, \delta_{y}^{x}, U_{i}^{n}\left(x_{1}, \cdots, x_{n}\right) \epsilon C$, if $\theta\left(y_{1}, \cdots, y_{m}\right)$, $\chi_{1}(\mathfrak{x}), \cdots, \chi_{m}(\mathfrak{x}) \epsilon C$, then $\theta\left(\chi_{1}(\mathfrak{x}), \cdots, \chi_{m}(\mathfrak{x})\right) \epsilon C$, and if $\rho(\mathfrak{x}, y) \epsilon C$ and $(\mathfrak{x})(E y)[\rho(\mathfrak{x}, y)=0]$, then $\epsilon y[\rho(\mathfrak{x}, y)=0] \epsilon C$.

Instead of the identity functions $U_{i}^{n}\left(x_{1}, \cdots, x_{n}\right)=x_{i}$, a second substitution schema $\theta\left(\chi_{1}(\mathfrak{x}), \chi_{2}(\mathfrak{y})\right)$ may be introduced.

Using the identity functions, we can replace substitutions which do not fall under the given schema by series of substitutions which do thus, $\theta(x, \phi(x, y, z), \psi(z, y))=\theta\left(U_{1}{ }^{3}(x, y, z)\right.$, $\left.\phi(x, y, z), \psi\left(U_{3}^{3}(x, y, z), U_{2}^{3}(x, y, z)\right)\right)$.

Definition 2. A relation $\epsilon C$ if its representing function $\epsilon C$.

Analogously to Gödel's I-IV :

I. The functions $C(x), S(x), U_{i}^{n}\left(x_{1}, \cdots, x_{n}\right) \epsilon C$. Every function (relation) obtained by substitution of functions which $\epsilon C$ for variables of functions (relations) which $\epsilon C \epsilon C$. If the relation $R(\mathfrak{x}, y) \epsilon C$ and $(\mathfrak{x})(E y) R(\mathfrak{x}, y)$, then $\epsilon y[R(\mathfrak{x}, y)] \epsilon C$.

II. If the relations $R, S \epsilon C$, so do $\bar{R}, R \bigvee S$ (hence also $R \& S$ ).

III. If the functions $\phi(\mathfrak{x}), \psi(\mathfrak{y}) \epsilon C$, so does the relation $\phi(\mathfrak{x})=\psi(\mathfrak{y})$.

IV. If the function $\phi(\mathfrak{x})$ and the relation $R(x, \mathfrak{y}) \epsilon C$, so do the relations $(x)[x<\phi(\mathfrak{x}) \rightarrow R(x, \mathfrak{y})]$ and $(E x)[x<\phi(\mathfrak{x}) \& R(x, \mathfrak{y})]$.

Proofs. $\quad C(x)=\epsilon y[x \cdot y=0]$. Let $\alpha(x)=\epsilon y\left[\delta_{y}^{x}=0\right]$. Then $\alpha(0)=1, \alpha(1)=0$. $S(x)=x+\alpha(C(x))$. For II we see that, if $\rho(\mathfrak{x})$ and $\sigma(\mathfrak{y})$ are the representing functions of $R$ and $S, \alpha(\rho(\mathfrak{x}))$ is the representing function of $\bar{R}$ and $\rho(\mathfrak{x}) \cdot \sigma(\mathfrak{y})$ is the representing function of $R \vee S$. Let $\gamma(x, y)=\alpha\left(\delta_{y}^{x}\right)$. Then $\gamma(x, x)=0$ and $\gamma(x, y)=1$ for $x \neq y$. III holds since $\gamma(\phi(\mathfrak{x}), \psi(\mathfrak{y}))$ is the representing function of $\phi(\mathfrak{x})=\psi(\mathfrak{y})$. In proof of IV, we have

$(x)[x<\phi(\mathfrak{x}) \rightarrow R(x, \mathfrak{y})] \equiv \epsilon x \overline{[R(x, \mathfrak{y})} \vee x=\phi(\mathfrak{x})]=\phi(\mathfrak{x})$, $(E x)[x<\phi(\mathfrak{x}) \& R(x, \mathfrak{y})] \equiv \overline{(x)}[x<\phi(\mathfrak{x}) \rightarrow \overline{R(x, \mathfrak{y})}]$.

V. $x<y \epsilon C . \quad x \equiv y(\bmod n) \epsilon C$.

* Kurt Gödel, Über formal unentscheidbare Sätze der Principia Mathematica und verwandter Systeme I, Monatshefte für Mathematik und Physik, vol. 38 (1931), pp. 173-198, Satz VII.

$\dagger$ Hilbert-Bernays, Grundlagen der Mathematik, vol. 1, pp. 412-421. 
For

$$
\begin{aligned}
x<y & \equiv \overline{(E z)}[z<x+1 \& x=y+z],[x \equiv y(\bmod n)] \\
& \equiv(E z)[z<x+y+1 \&\{x=y+z \cdot n \vee y=x+z \cdot n\}],
\end{aligned}
$$

(see Gödel, p. 191).

VI. If the relation $x_{0}=\phi(\mathfrak{x}) \epsilon C$, the function $\phi(\mathfrak{x}) \epsilon C$.

For $\phi(\mathfrak{x})=\epsilon x_{0}\left[x_{0}=\phi(\mathfrak{x})\right]$.

VII. If $\phi(\mathfrak{x})$ is a primitive recursive function, the relation $x_{0}=\phi(\mathfrak{x}) \epsilon C$.

The proof is by induction with respect to the number of applications of schemas (1) and (2) in the primitive recursive definition of $\phi(\mathfrak{x})$ (see Kleene, Def. 1). First, the proposition $x_{0}=\phi(\mathfrak{x}) \epsilon C$ holds if $\phi(\mathfrak{x})$ is one of the functions $S(x), C(x)$, $U_{i}^{n}\left(x_{1}, \cdots, x_{n}\right)$, by I and III. Second, if it holds for $\theta\left(y_{1}, \cdots, y_{m}\right), \chi_{1}(\mathfrak{x}), \cdots, \chi_{m}(\mathfrak{x})$, then $\chi_{1}(\mathfrak{x}), \cdots, \chi_{m}(\mathfrak{x}) \epsilon C$ by VI, and it holds for $\theta\left(\chi_{1}(\mathfrak{x}), \cdots, \chi_{m}(\mathfrak{x})\right)$ by I. Third, suppose Gödel, p. 192, 2 holds, where $\psi\left(x_{2}, \cdots, x_{n}\right), \mu\left(x_{1}, \cdots, x_{n+1}\right)$ have the property in question. Following Gödel's discussion, Hilfsatz 1 holds with $n>d$, since $d$ is determined first, and $n$ is any number which satisfies the congruences $n \equiv f_{i}[\bmod (1+(i+1) d)]$, $(i=0, \cdots, k-1)$. Again, $x=[n]_{p} \in C$. If $P\left(x_{0}, \cdots, x_{n}\right) \equiv x_{0}$ $=\phi\left(x_{1}, \cdots, \quad x_{n}\right), \quad S\left(x_{0}, x_{2}, \cdots, x_{n}\right) \equiv x_{0}=\psi\left(x_{1}, \cdots, x_{n}\right)$, $T\left(x_{0}, \cdots, x_{n+1}\right) \equiv x_{0}=\mu\left(x_{1}, \cdots, x_{n+1}\right)$, the relation on page 193 can be restated as follows: $P\left(x_{0}, \cdots, x_{n}\right) \equiv x_{0}=[N]_{1+D\left(x_{1}+1\right)}$, where $N$ stands for $\epsilon n\left[(E d)\left[d<n \&\left\{S\left([n]_{d+1}, x_{2}, \cdots, x_{n}\right)\right.\right.\right.$ $\left.\left.\left.\&(k)\left[k<x_{1} \rightarrow T\left([n]_{1+d(k+2)}, k,[n]_{1+d(k+1)}, x_{2}, \cdots, x_{n}\right)\right]\right\}\right]\right]$ and $D$ stands for $\epsilon d\left\{S\left([N]_{d+1}, x_{2}, \cdots, x_{n}\right) \&(k)\left[k<x_{1} \rightarrow T\left([N]_{1+d(k+2)}\right.\right.\right.$, $\left.\left.\left.k,[N]_{1+d(k+1)}, x_{2}, \cdots, x_{n}\right)\right]\right\}$. It follows that $P\left(x_{0}, \cdots, x_{n}\right) \epsilon C$.

VIII. Every primitive recursive function $\epsilon C$ (by VI, VII).

IX. Every recursive function $\epsilon C$ (by VIII, I, Kleene IV).

$\mathrm{X}$. Every function which $\epsilon C$ is recursive.

This follows from the recursiveness of $x+y, x \cdot y, \delta_{y}^{x}$ and functions obtained from recursive functions by substitution, and Kleene V. Combining IX and $\mathrm{X}$, we have the following result.

$\mathrm{XI}$. The class $C$ of functions is identical with the class of recursive functions.

The UNIVERsity OF Wisconsin 